\title{
MAKING TRANSPORT SAFER: V2V-BASED AUTOMATED EMERGENCY BRAKING SYSTEM
}

\author{
Vicente Milanés ${ }^{1}$, Enrique Onieva ${ }^{2}$, Joshué Pérez ${ }^{3}$, Javier Simó ${ }^{4}$, \\ Carlos González ${ }^{5}$, Teresa de Pedro ${ }^{6}$ \\ 1, 2, 3, 5, 6 AUTOPIA program, Centre for Automation and Robotics \\ (CAR) La Poveda-Arganda del Rey, 28500 Madrid, Spain \\ ${ }^{4}$ Signal Theory \& Communications Department, Universidad Rey Juan Carlos, \\ Camino del Molino s/n, 28943 Fuenlabrada, Madrid, Spain \\ E-mails: ${ }^{1}$ vicente.milanes@csic.es (corresponding author); ${ }^{2}$ enrique.onieva@csic.es; \\ 3joshue.perez@csic.es; ${ }^{4}$ javier.simo@urjc.es; ${ }^{5}$ carlos.gonzalez@csic.es; ${ }^{6}$ teresa.pedro@csic.es
}

Received 6 October 2010, accepted 31 July 2011

\begin{abstract}
An important goal in the field of intelligent transportation systems (ITS) is to provide driving aids aimed at preventing accidents and reducing the number of traffic victims. The commonest traffic accidents in urban areas are due to sudden braking that demands a very fast response on the part of drivers. Attempts to solve this problem have motivated many ITS advances including the detection of the intention of surrounding cars using lasers, radars or cameras. However, this might not be enough to increase safety when there is a danger of collision. Vehicle to vehicle communications are needed to ensure that the other intentions of cars are also available. The article describes the development of a controller to perform an emergency stop via an electro-hydraulic braking system employed on dry asphalt. An original V2V communication scheme based on WiFi cards has been used for broadcasting positioning information to other vehicles. The reliability of the scheme has been theoretically analyzed to estimate its performance when the number of vehicles involved is much higher. This controller has been incorporated into the AUTOPIA program control for automatic cars. The system has been implemented in Citroën C3 Pluriel, and various tests were performed to evaluate its operation.
\end{abstract}

Keywords: road traffic, traffic accident, traffic safety, motor vehicle, collision avoidance, wireless communications.

\section{Introduction}

The trend towards future road transport is a move from simple driving aids to automatic driving controls. The goal is to attempt to reduce the number of collisions, or at least to mitigate their consequences (Prentkovskis et al. 2010a, 2010b; Sokolovskij et al. 2007; Černiauskas et al. 2010). Factors such as poor visibility of other users, not maintaining a safe distance, or ignoring traffic signals are some of the main causes of accidents (Use of Intelligent... 2006). Most of these causes can be associated with excessive or inappropriate speed. In fact, a third of fatal or serious accidents are due to this reason. Considering different solutions to the above proposed problems, the development of autonomous vehicles is a particularly open field of discussions today.

Research on developing automated vehicles to improve the safety and efficiency of highways or freeways is indeed one of the most extensively studied topics in the field of intelligent transportation systems (ITS) that can be divided into two areas one of which is centred on roadway infrastructure, and its primary aim is to add information and communications technology to make driving easier (Junevičius, Bogdevičius 2009; Çalışkanelli et al. 2009; Boumediene et al. 2009). The other is centred on vehicles, and its goal is to develop driving assistance that reduces dependence on the driver (Prentkovskis et al. 2010a, 2010b; Skrickij, Bogdevičius 2010; Grīslis 2010; Sapragonas, Dargužis 2011). The present work falls into the second category, specifically on reducing the likelihood of collision by means of an emergency braking system based on vehicle to vehicle communications $(\mathrm{V} 2 \mathrm{~V})$.

Different manufacturers have installed driver assistance systems in their cars to prevent accidents. In early 2008, Ford made public its radar technology based on adaptive cruise control (ACC) fitted behind the front grille. This system allows the driver to select one of five different time gaps of separation from the preceding car. 
In a parallel line of work, Toyota has developed a broader-range radar sensor with an operation based on applying smooth braking or acceleration to maintain a safe distance up to the pre-set speed. However, these systems have two main drawbacks. First, those based on radar technology have problems with curved stretches where the rear end of the preceding car is lost. Second, they all apply smooth pressure onto the brake and throttle pedals, and therefore no hard braking is possible.

Bearing this in mind, we propose $\mathrm{V} 2 \mathrm{~V}$ communication improvement to ACC systems in order to avoid radar failures in the curved stretches of the road, so that together with a specific system to permit hard braking when a sudden stop of the preceding car is detected.

$\mathrm{V} 2 \mathrm{~V}$ communications for collision warning systems (CWS) have been developed using differential global positioning systems (DGPS) (Tan, Huang 2006). However, good automation of the throttle pedal, and above all the brake pedal, is mandatory in order to extend such prevention to autonomous systems capable of avoiding collisions.

Assistance systems that warn the driver of imminent collisions through visual or audible signals have been included in commercial cars in recent years. Although these techniques are necessary and provide a warning to the driver, the dependence on human reaction time regarding a decision to be made remains a problem. For this reason, our proposal involves direct action on car actuators. As far as the controllers are concerned, those associated with longitudinal control, in particular with the brake, bear the main responsibility for reducing the likelihood of crashes. Risk situations such as blind curves, crossroads with poor visibility, or unexpected braking of the preceding car suggest that an emergency system associated with the brake pedal is needed to avoid accidents.

There have been various approaches to the automation of the brake pedal. Anwar (Anwar, Zheng 2007) describes a nonlinear sliding-mode-type controller for slip regulation in a braking event for a car equipped with an electromagnetic brake-by-wire (BBW) system. Xiang et al. (2008) focuses on fault tolerance design and vehicle braking control schemes for electromechanical brake systems. Naranjo et al. (2006) uses an encoder coupled to a DC motor in the original circuit to obtain an automatic braking system pulling on the brake pedal. Prohaska and Devlin (1998) uses hydro-boost power brake servo modified for external control. Kim et al. (1996) develops a fuzzy controller to actuate braking. Gerdes and Hedrick (1997) uses a brake vacuum booster with a separated actuator mounted between the booster and master cylinder. Finally, pneumatic braking controllers have been developed for other transportation vehicles such as buses (Bu, Tan 2007) and trains (Zhuan, Xia 2007).

The paper describes a control system that implements ACC fuzzy controller extension consisting of emergency stopping applied via an electro-hydraulic braking system. The autonomous system is implemented as an independent braking circuit that can work in parallel with the original circuit. We designed a controller to carry out the stopping process such that, when an emergency situation is detected, the controller deactivates the fuzzy ACC controller and initiates a precision emergency stop. The least squares method used by other researchers (Zhou, Mahmassani 2006; Venture et al. 2006) is applied to experimentally determine the relationships between different variables of the system.

Experiments were performed to study the behaviour of the system in a realistic situation, i.e. sudden, unexpected braking of the preceding car. In urban traffic, this situation is the cause of many accidents either from colliding with other cars or hitting nearby pedestrians as a result of making abrupt steering manoeuvres.

We also propose a V2V communication system based on broadcasting positioning information over WiFi. Each vehicle estimates its own position and speed based on the information gathered through DGPS system, and then distributes this information to other vehicles in the vicinity. The majority of communication problems associated to V2V communications are successfully addressed in upcoming standards IEEE 802.11p, Dedicated Short-Range Communication (DSRC) and IEEE 1609 , including reserved frequencies, differentiation of traffic classes, security and multi-channel operation (IEEE Std 802.11:2007; IEEE P802.11p/D3.0:2007; Uzcategui, Acosta-Marum 2009; Jiang, Delgrossi 2008). However, in this case, the critical nature of an emergency breaking system requires the evaluation of the performance and reliability of 802.11 Medium Access Control. Based on this analysis, an ad-hoc mechanism is proposed to reduce failure probability to an acceptable level. IEEE $802.11 \mathrm{p}$ behaviour has been successfully emulated in experiments using 802.11a PHY + 802.11e MAC and adjusting some MAC parameters to the appropriate values. The use of the $5.8 \mathrm{GHz}$ band in our test bed assures that our results are valid for DSRC band (Sen, Matolak 2008; Tufail et al. 2008; Iyer et al. 2008).

\section{Equipment of the Prototype Vehicle}

This section describes the systems installed in the car. The vehicle is one of the fleet of AUTOPIA program the goals of which are to implement automatic driving using real, mass-produced vehicles tested on real roads and to develop automated systems using modular components that can be immediately applied in automotive industry.

The main sensor inputs of the system are an inertial measurement unit (IMU) and a high precision global positioning device. When using these, the system controls vehicle-driving actuators, i.e. steering and throttle and brake pedals. The vehicle includes an on-board PCbased computer, real-time centimetre kinematic DGPS (RTK-DGPS), wireless LAN support, a pulse-width modulation (PWM) controller to actuate the steering wheel, a digital/analog I/O card for controlling the throttle and an electro-hydraulic system for braking. Fig. 1 shows the structure of the control system that manages all these devices. 


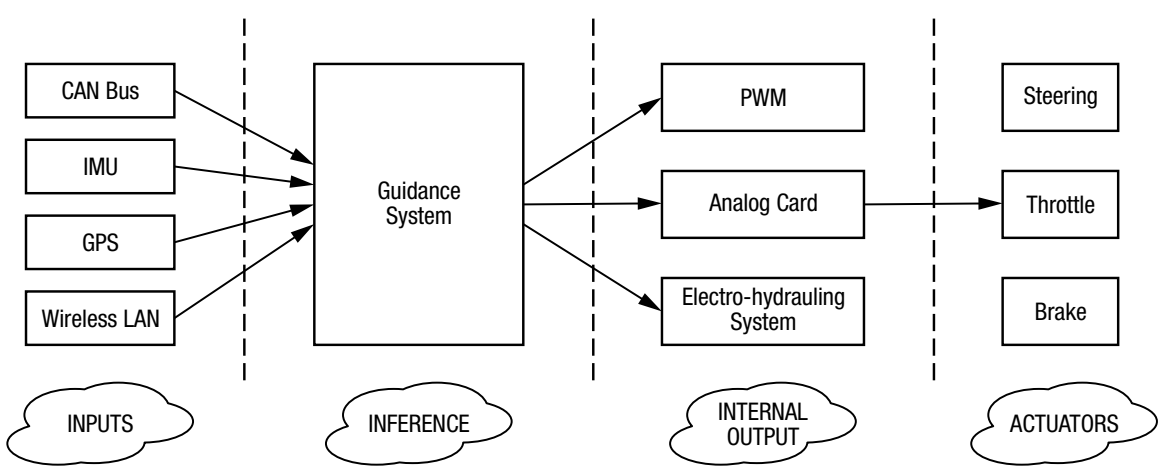

Fig. 1. The structure of controlling AUTOPIA system

Guidance is carried out by a cooperative system based on the combination of GPS positioning and an inertial measurement unit (IMU) plus odometer positioning in case the GPS signal is lost (Milanés et al. 2008). This system is modelled using fuzzy variables and rules to follow the trajectory marked by high-precision digital cartography defining the target route. In addition to the steering wheel and vehicle speed functionalities, the system also considers variables that can be used for adaptive cruise control (ACC).

The system is mounted on a Citroën C3 Pluriel car (Fig. 2) and three automatic-drive switches to activate controllers associated with the throttle, brake and steering wheel are installed in the dashboard.

\section{Electro-Hydraulic Braking System}

The main aim at developing the automatic braking system was to achieve an independent braking circuit added onto the original circuit. Two shuttle valves are installed and connected to the input of the antilock braking system (ABS) in order to keep two circuits independent. Each valve permits flow from either of two inlet ports to a common outlet by means of a free-floating metal ball that shuttles back-and-forth according to relative pressures at two inlets. One of the inlets is connected to the electro-hydraulic braking system and the other one - to the original one. These valves allow only the input with larger pressure to provide supply to calipers, and thus permit the two braking systems to cooperate, but independently of each other.

The hydraulic system designed to connect to the shuttle valves consists of a one-litter capacity brake fluid

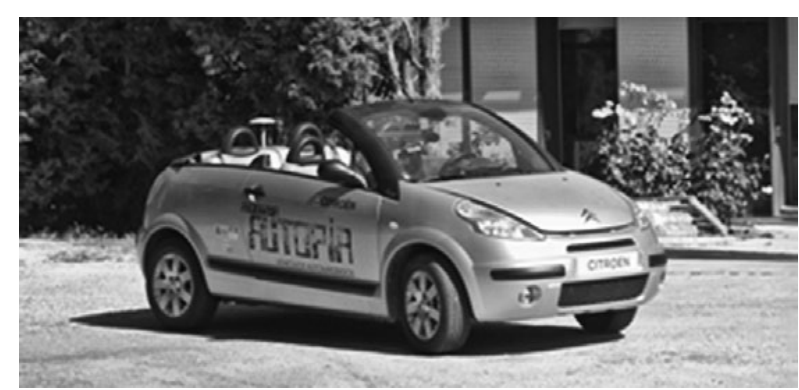

Fig. 2. Autonomous Citroën C3 Pluriel tank that includes a gear pump and coupling to a $12 \mathrm{~V}$ $350 \mathrm{~W}$ DC electric motor. In the experiment, the maximum pressure supplied by the brake pedal was measured. A pressure limiter tube set at 120 bars is installed in the system to avoid damage to the circuits. Two more valves were installed to control the system: a voltagecontrolled electro-proportional pilot regulated the applied pressure and a spool directional valve controlled the activation of the electro-hydraulic system by means of a digital signal. A schematic outline of the electrohydraulic system design is shown in Fig. 3.

\section{Control Architecture}

This section presents the control system to perform emergency braking. To this end, decision units to choose among different controllers in the function of traffic situations are presented. Next, a fuzzy controller to carry out ACC manoeuvres is shown. Finally, the emergency braking algorithm is described.

\subsection{Decision Unit}

The design includes three different fuzzy controllers to perform automatic driving (see Fig. 4). First, denominated 'normal driving' is designed to follow a target route the values of which in UTM coordinates together with reference speed are loaded from a database. This automatic mode is running when no vehicles are detected in the surrounding area. Second, denominated 'ACC + stop \& go driving' is capable of detecting a preceding car and adjusting its own speed appropriately. The third fuzzy controller, denominated 'overtaking', is designed to perform the overtaking manoeuvre on two-way roads

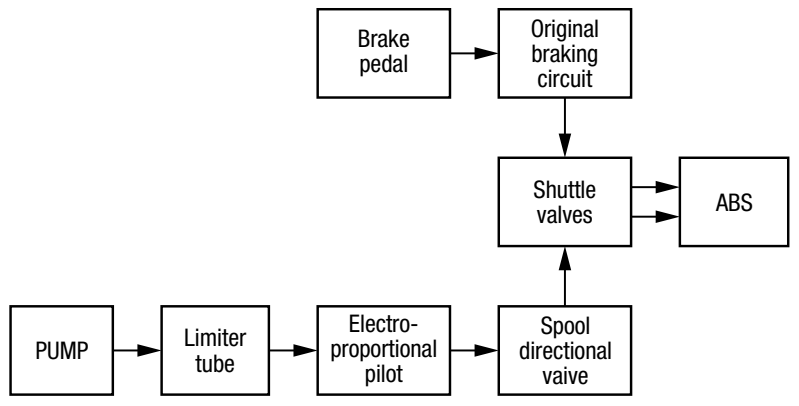

Fig. 3. The design of the electro-hydraulic braking system 


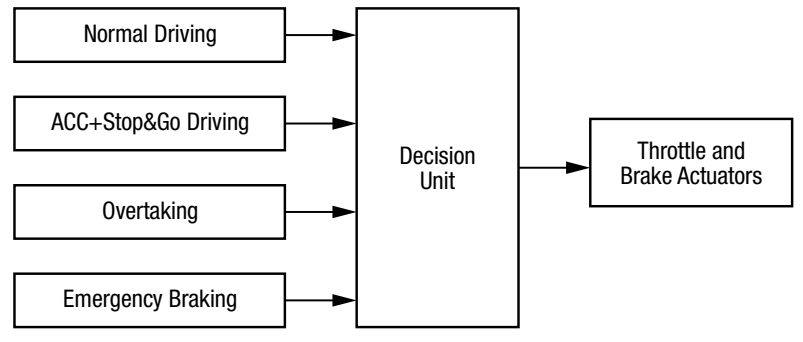

Fig. 4. The architecture of the control system

without traffic in the opposing lane. Finally, we developed a new controller, denominated 'emergency braking', for a special case the priority in which is avoiding an imminent crash.

A decision unit is responsible for choosing an appropriate controller to actuate on the throttle and braking pedals in each control cycle. This unit takes into account variables corresponding to the vehicle itself and to any other vehicles in the surrounding area.

It must be emphasized that the last 'emergency braking' controller is not a fuzzy one as when it takes over, a high risk situation is detected. Then, the only action to be followed is to stop the car as soon as possible. In this case, there are two prerequisites for it to be activated: first, information from another car must be received via $\mathrm{V} 2 \mathrm{~V}$ communication; second, sharp deceleration together with a dangerously short distance from the preceding car must be detected.

\subsection{ACC Fuzzy Controller}

AUTOPIA program implemented the ACC system in an electric Citroen Berlingo van (Naranjo et al. 2006). This controller, based on fuzzy logic (Djukić et al. 2010), was adapted for gasoline Citroën C3 Pluriel used in the experiments described above. Adaptation consisted of modifying the linguistic values of the fuzzy rules to take into account the characteristics of the new vehicle. The time gap is the period of time that the trailing car would take to cover the distance from its actual position to the leading car's position; the derivatives of the time gap were used as input values. Actuation on the throttle and brake are generated as output values.

Fig. 5 illustrates the behaviour of the ACC fuzzy controller system. The upper plot shows the speeds of the two cars, the black curve represents the leading vehicle and the grey curve - the trailing one. The middle plot shows the distance separating the two cars and the lower plot - the acceleration of the leading car.

During this trial, the leading vehicle was driven manually. This can be noted in speed fluctuations. Following approximately $40 \mathrm{~s}$, sudden sharp deceleration is well accepted by the system. However, at the end, following $72 \mathrm{~s}$, an even stronger deceleration of the leading vehicle due to sudden hard braking is not at all appropriately responded to the system. Indeed, one observes in the distance plot presented in the figure that the values become negative, thus indicating that the trailing car would have crashed into the leading car. All the experiments described in this paper were carried out using sufficient lateral displacement between the cars to avoid an actual crash in case of failure.

Hence, it is clear that the ACC system that automatically reduces the speed of the car in case it is moving too close to the leading vehicle or increases it in case it is too far away is not designed to respond to abrupt changes in speed. Therefore, a new robust and safe system to resolve this kind of circumstance should be helpful.
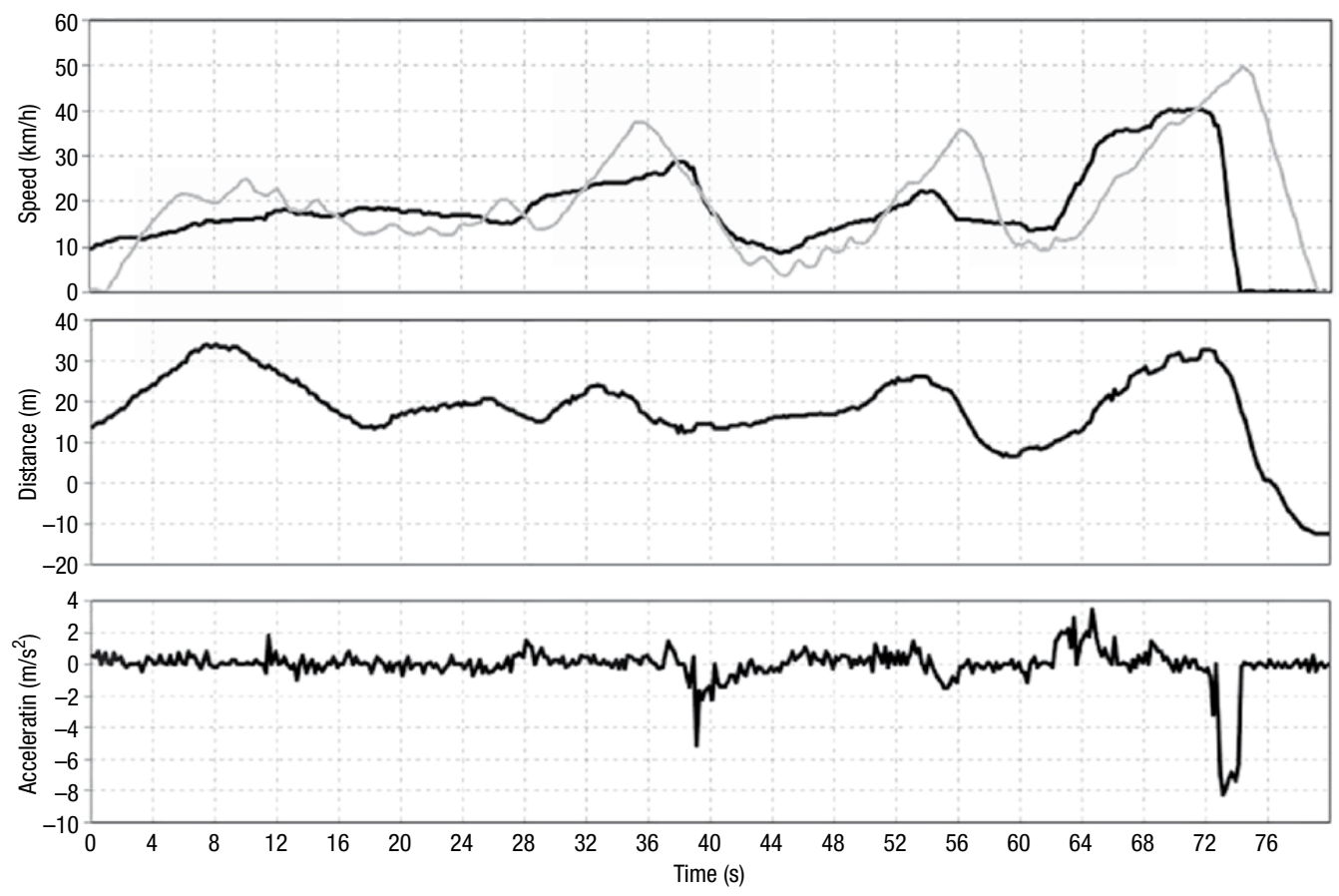

Fig. 5. ACC trial with emergency braking of the leading vehicle 


\subsection{Emergency Braking Module}

This subsection presents a proposal for how to deal with the problem of sudden hard braking. In our opinion, the essential aspects of the proposal are not limited to ACC extension; nevertheless, the system is applicable to manual driving in the case that an imminent crash is detected.

An emergency braking controller must compromise between avoiding a collision and ensuring the integrity of passengers. Too sudden braking can cause a chain of collisions involving nearby vehicles. For this reason, we implemented a system based on both the distance to collision and the speed of the car. The procedure followed was divided into two steps. The first one was to experimentally determine the function:

\section{Braking Pressure $=f($ distance, speed $)$}

and the second - to determine the actual braking algorithm. The least squares method was used for determining the functional relationship of the variables involved in control: distance covered, speed and braking pressure. The distance covered is obtained from RTK-DGPS the accuracy of which is around two centimetres and speed is taken from the CAN bus. The sampling rate is $5 \mathrm{~Hz}$ fixed by GPS.

The relationship between the voltages applied to the electro-proportional pilot and pressure actually applied to the wheels is non-linear. Experiments were performed to obtain the function representing the behaviour of this part of the system. The experiments consisted of measuring the distance that the car covers for the sets of two fixed parameters: the initial speed set at around fifty kilometres per hour, speed limit in urban areas and braking pressure that varied in the steps of $0.5 \mathrm{~V}$ from $0 \mathrm{~V}$ at which the pump exerts the minimal pressure on the wheels, to $4.5 \mathrm{~V}$ which corresponds to the maximum pressure bearable by the wheels due to the limits imposed by the mechanical system. The resulting ten plots are shown in Fig. 6.

The maximum distance required to completely stop the car was $80 \mathrm{~m}$ and the minimum distance $11.7 \mathrm{~m}$. Tests attempting to stop the car in a distance of less than $11.7 \mathrm{~m}$ were unsuccessful because the wheels were blocked by the anti-lock braking system. These experiments yielded the relationship between the variables, and then, the least squares method was used for determining control surface giving pressure to apply for an emergency stop in critical situations. This surface (Fig. 7) was validated up to the limit imposed by the maximum braking force and minimum distance, with results showing the system to present good behaviour.

The second step in implementing an emergency stop to avoid collisions was to apply braking force in real time. The system is based on the distance to the preceding car and the speed of the car. When activated, it determines pressure to exert on the wheels as a function of that distance and speed recalculating the distance and appropriately adjusting pressure to apply in each control cycle.

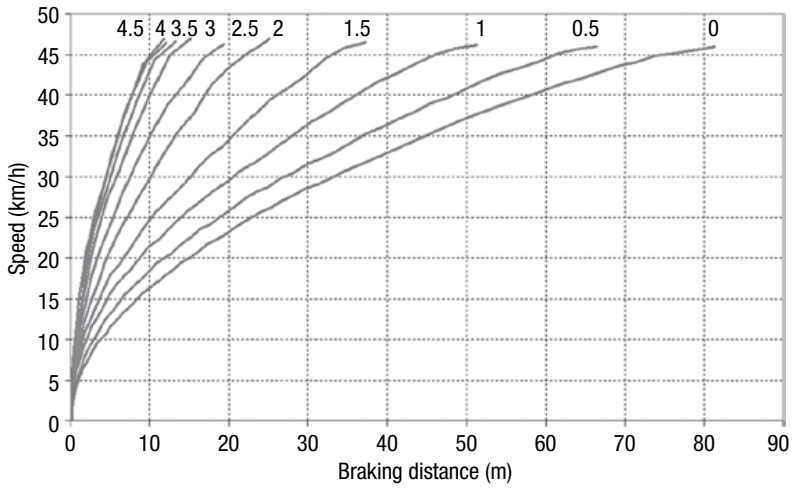

Fig. 6. Relationship between speed and braking distance for different values of braking pressure (in volts applied to the electro-proportional pilot)

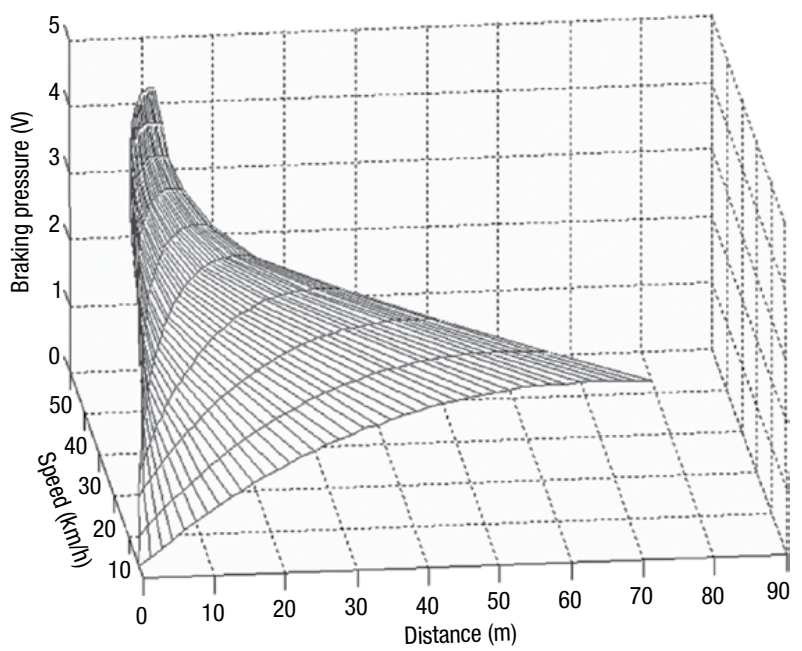

Fig. 7. Control surface obtained applying the least squares method

The activation of the braking system is based on the measurement of two variables: the distance between the cars and acceleration of the leading vehicle. The reason for using both these variables is that each of those is insufficient. For instance, sharp deceleration will not be dangerous if there is sufficient distance separating vehicles; similarly, a short separation distance will not be dangerous if vehicles are moving very slowly. For the system to be activated the first condition is when the deceleration of the leading vehicle surpasses $7 \mathrm{~m} / \mathrm{s}^{2}$ (Amodeo et al. 2010) - only dry asphalt is considered in this work. Then, if the distance falls below $15 \mathrm{~m}$ and results from adding minimum distance $(11.7 \mathrm{~m})$, DGPS errors $(0.52 \mathrm{~m}), \mathrm{V} 2 \mathrm{~V}$ maximum error (see below) at $50 \mathrm{~km} / \mathrm{h}$ $(1.06 \mathrm{~m})$ and safety distance $(1.72 \mathrm{~m})$, emergency brake itself is activated.

\section{Vehicle to Vehicle Communication}

\subsection{Analysis of Communication Issues}

The core of this work is the suggested emergency breaking system. Communications play an instrumental role in our research. However, due to the critical nature of 
the system proposed, we need to prove that communications give reliable support to it. As briefly explained in the introduction of this paper, emergent IEEE 802.11p, IEEE 1609 and DSRC standards solve several issues of V2V communications, including security, access differentiation and a dedicated frequency band that is particularly important. However, even if a service channel is dedicated to the $\mathrm{V} 2 \mathrm{~V}$ exchange of positioning information, it is not obvious whether Medium Access Control may ensure reliable V2V communications for such a critical system. This section analyzes communication issues specific to our case and propose a practical solution to broadcasting information reliably. The only purpose of the introduced mechanism is to demonstrate that IEEE 802.11 can be successfully used for providing reliable $\mathrm{V} 2 \mathrm{~V}$ communications as required in our emergency breaking system. More efficient mechanisms may be suggested and better communication strategies must be found for a more general case when communication systems must be shared for other services. However, these issues go beyond the scope of this work.

$\mathrm{V} 2 \mathrm{~V}$ communications must take into account the dynamic nature of traffic. Relative positions, speeds and distances among vehicles change very fast. In such context:

- A vehicle must know about the position and speed of several other vehicles in its neighbourhood. Hence, such information must be broadcasted from each vehicle to all neighbours under a given critical distance. However, a broadcast packet in 802.11 will never be confirmed, and a vehicle never knows whether a given neighbour has received the packet or not. This also means that collisions will not be detected and automatic retransmissions will never happen due to collisions.

- The well-known problem of the hidden node will be generally present in any V2V scenario. Therefore, a significant probability that a vehicle can receive information from two neighbours that cannot listen to each other always exists. This makes useless two strategies that 802.11 uses for reducing collision probability: physical carrier detection and NAV (network allocation vector) used in combination with RTS/CTS mechanism.

- Control cycles in all vehicles at a given area receive positioning information from satellites simultaneously through their DGPS systems, hence causing implicitly a high level of synchronization. In such conditions, broadcasting packets as soon as a vehicle extracts information from the DGPS system would imply an extremely high probability of collision. In order to lower collision probability, the instant chosen for transmitting broadcast packets within control cycle must be stochastic.

- For a number of vehicles in the vicinity as high as $N=40$, even with previous consideration, the collision probability of a single broadcast packet would be higher than $p>0.02$, which would be unacceptable for service as critical as an emergency breaking system.

\subsection{Reliability of the V2V Communications System}

The obvious consequence of the previous considerations is the need of transmitting several broadcast packets per control cycle from each vehicle, choosing stochastically the instants of transmission and updating the information contained in each packet as much as possible because nobody knows what packets will be successfully received and what will collide. Next, we need to find out the optimal value of the number of packets transmitted per control cycle, the lowest error probability and the exact mechanism for its implementation.

The proposed scheme is built on the top of custom IEEE 802.11e cards working in the band of $5.8 \mathrm{GHz}$. The cards and drivers have not been modified at all apart from some variable MAC parameters that are adjusted following the values displayed in Table 1 and recommendations of IEEE 1609.4 standard for the major priority service class. The proposed broadcasting scheme is programmed on the top of the driver as a user-space program. All vehicles obtain information on precise positioning through DGPS and broadcast it several times per control cycle. The description of this mechanism follows. Collision probability may be dramatically improved only by slotting the channel, which is especially easy in our case because the size of all the packets exchanged by our system will have a constant well-known size. The reception of DGPS information determines the starting point of control cycles in all vehicles. A control cycle is divided into $K$ high-level slots so that transmissions can only happen at the beginning of any slot. In such conditions, if slot duration is long enough to contain the complete transmission of a packet plus round-trip propagation time to the farthest station in our communication range, collision probability will be minimized.

The proposed broadcast packet contains four fields: time-stamp, UTM latitude, UTM longitude and speed implemented as one unsigned long integer of 4 bytes, two double real numbers of 8 bytes each and one 'float' of 4 bytes respectively, totalling 24 bytes. We will work at a fixed speed of $6 \mathrm{Mbps}$. The obtained results could be improved by working at higher speeds in case of favourable conditions for channel propagation. The values of different parameters that must be considered in calculations are included in Table 1.

It should be noticed that a $160 \mu$ s high-level slot size accomplishes the condition given to optimize collision probability under our conditions. Thus, by dividing control cycle in 1250 slots at the control level and imposing that packets are transmitted stochastically at the beginning of any high-level slot, we will have the lowest possible collision probability. The IEEE 802.11 contention window would never be used in our scenario because the channel would always be free at the beginning of a high-level slot. If traffic is present in the same band from other sources, more accurate analysis should be performed. 
Table 1. Medium access control parameters used in calculations and experiments. IEEE 802.11a has been used and considered for compatibility with available experimental materials, but adjustments to IEEE 802.11 p would be insignificant

\begin{tabular}{ll}
\hline SIFS & $16 \mu \mathrm{s}$ \\
\hline Bitrate & $6 \mathrm{Mbps}$ \\
\hline SlotTime & $9 \mu \mathrm{s}$ \\
\hline DIFS & $34 \mu \mathrm{s}$ \\
\hline Round-trip propagation time & $2 \mu \mathrm{s}$ \\
\hline PLCP Overhead (preamble and header) & $52 \mu \mathrm{s}$ \\
\hline MAC Overhead (header) & $37.33 \mu \mathrm{s}$ \\
\hline Payload & $32 \mu \mathrm{s}$ \\
\hline Total packet duration & $121.67 \mu \mathrm{s}$ \\
\hline DIFS+broadcast & 157.33 \\
\hline Control cycle & $200 \mathrm{~ms}$ \\
\hline Proposed high-level slot size & $160 \mu \mathrm{s}$ \\
\hline Number of high-level slots per control cycle & 1250 \\
\hline
\end{tabular}

A total number of $m$ broadcast packets will be sent per control cycle. It has already been mentioned that $m=1$ would give a very poor result. As we increase $m$, one can suspect that the probability of one of the packets to be successfully received by a neighbour station is initially increased. It is also straightforward to see that an extremely high value of $m$ would be also suboptimal. Hence, the optimal value of $m$ that minimizes error probability must be found. Note that the correct transmission and reception of at least one packet per control cycle will be considered success; even though $(m-1)$ other packets in the same control cycle may have collided. Consequently, we define the probability of failure $\left(P_{f}\right)$ as the probability of all $m$ packets broadcasted during control cycle to collide.

We have defined $K$ as the number of high-level slots in control cycle. We are proposing that variable to have constant value $K=1250$, but keep it in the analytical expressions to make our analysis as general as possible. $m$ packets are sent in randomly chosen slots. The slot for the first transmission is chosen within $K$ possible slots using a uniform random variable; then the slot for the second transmission will be chosen within $K-1$ free slots using the same procedure etc.

The probability of a vehicle transmitting a packet at any given high-level slot is given in Equation 1:

$$
\tau=\frac{m}{K} \text {. }
$$

The conditional probability of the collision of any packet supposing that it has already been transmitted would be given by Equation 2:

$$
p=1-(1-\tau)^{N-1} \text {. }
$$

Hence, the probability of communication failure in control cycle corresponds to the probability of the collision of $m$ packets and is provided in Equation 3:

$$
P_{f}=p^{m}=\left(1-(1-\tau)^{N-1}\right)^{m}
$$

Failure probability in one cycle $\left(P_{f}\right)$ is represented in Fig. 8 and that during two consecutive control cycles is shown in Fig. 9. Due to high collision probability, we consider that error probability due to propagation issues can be neglected.

Fig. 10 represents the optimum value of $m$ that minimizes the probability of failure depending on the number of vehicles within the communication range. Table 2 gives optimal $m, P_{f}, P_{f^{2}}$ (probability of fail-

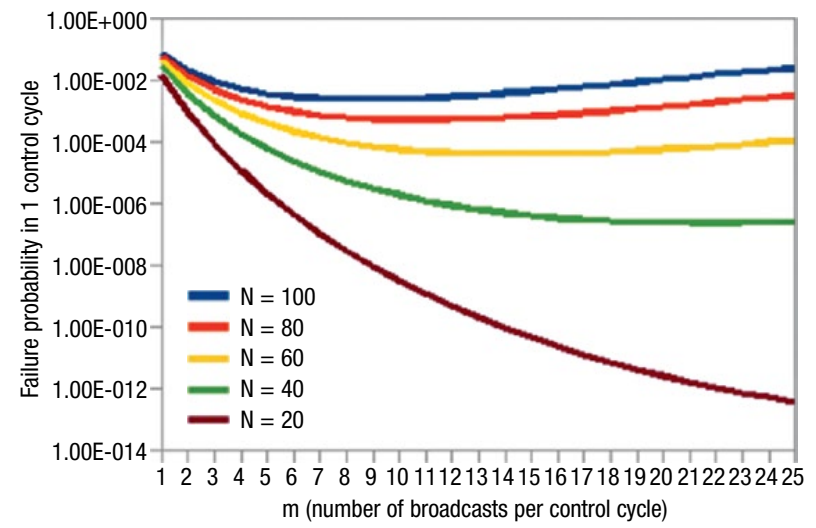

Fig. 8. The probability of communication failure in one control cycle

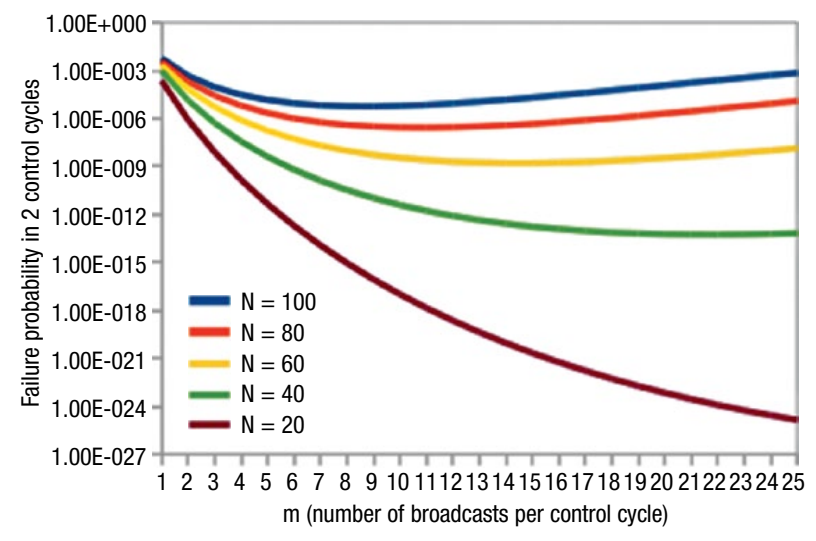

Fig. 9. The probability of communication failure in two control cycles

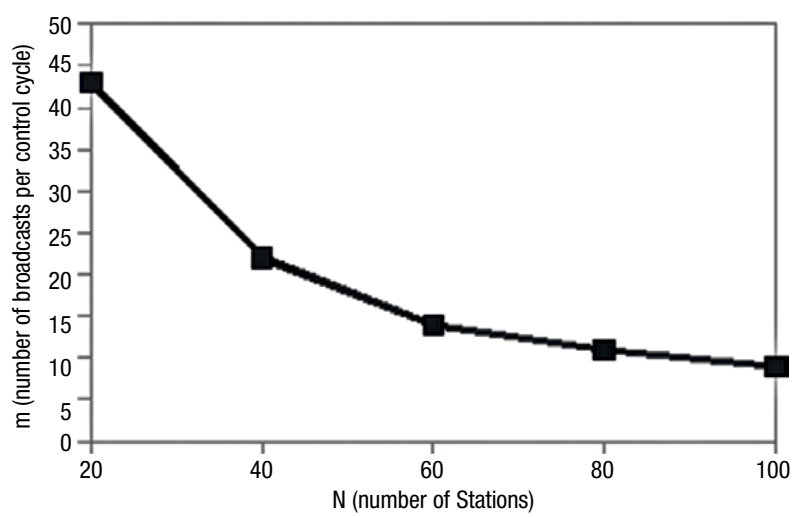

Fig. 10. Optimum $m$ as a function of $N$ 
Table 2. Failure probability of the communication system and medium time between failures

\begin{tabular}{ccccc}
\hline $\begin{array}{c}\text { Number of } \\
\text { neighbour } \\
\text { vehicles }\end{array}$ & $\begin{array}{c}m \text { that } \\
\text { minimizes } \\
P_{f}\end{array}$ & $\begin{array}{c}\text { Minimum } \\
P_{f}\end{array}$ & $\begin{array}{c}\text { Minimum } \\
P_{f}^{2}\end{array}$ & MTBF (h) \\
\hline 20 & 43 & $3.29 \cdot 10^{-14}$ & $1.08 \cdot 10^{-27}$ & $5.13 \cdot 10^{22}$ \\
\hline 40 & 22 & $2.35 \cdot 10^{-7}$ & $5.52 \cdot 10^{-14}$ & $1.01 \cdot 10^{9}$ \\
\hline 60 & 14 & $4.04 \cdot 10^{-5}$ & $1.63 \cdot 10^{-9}$ & $3.40 \cdot 10^{4}$ \\
\hline 80 & 11 & $5.16 \cdot 10^{-4}$ & $2.66 \cdot 10^{-7}$ & $2.09 \cdot 10^{2}$ \\
\hline 100 & 9 & $2.38 \cdot 10^{-3}$ & $5.66 \cdot 10^{-6}$ & 9.81 \\
\hline
\end{tabular}

ure in two consecutive control cycles) as well as MTBF (Mean Time Between Failures) calculated taking into account the probability of failure in two consecutive control cycles.

Failure probability that is lower than $10^{-6}$ per control cycle can be achieved for less than 45 vehicles within the range of communication. When doubling the number of vehicles, failure probability for two consecutive control cycles would be the same. On the other hand, distances among vehicles are naturally increased as speed becomes higher, which means that the system will be more reliable at higher speeds because the maximum number of vehicles within the range of communications will be lower. In order to estimate if those failure probabilities are low or high, one must think that communication failure will have a real impact only if:

- it affects the reception of positioning information from the immediate preceding vehicles;

- it happens when a sudden deceleration occurs in those vehicles.

The joint probability of those three events is practically equal to null. However, the system can determine when failure is likely to happen by computing the number of detected collisions. In that case, alert can be issued to the driver to inform that the system is temporarily unreliable

In any real scenario, for optimal results, the number of neighbour's vehicles that can be detected should be kept under forty $(N<40)$. A very simple collaborative strategy to achieve the required result is the adaptation of transmission power depending on the number of vehicles detected during one or two control cycles.

The number of broadcast packets may also be adjusted to its optimal value dynamically depending on the number of neighbour's vehicles detected.

\subsection{Implementing a Subsystem of Communications}

The control cycle of the system has a $200 \mathrm{~ms}$ period that starts when the DGPS system provides updated information about position, speed and time. The initial error of that information is lower than $50 \mathrm{~cm}$, which is fairly good for our purposes. However, we intend to send $m$ broadcast packets containing that information during control cycle. Speed information may be updated every $40 \mathrm{~ms}$ along with data obtained from the CAN bus. Time can also be updated for each packet using system clock. The position can be estimated using time, position and speed at the beginning of control cycle and updated time and speed. Each of $m$ broadcast packets in control cycle is supposed to contain updated information, not only a copy of the first version.

Initially $m=20$, and transmission power will be set to maximum. Control cycle will take the following actions concerning $\mathrm{V} 2 \mathrm{~V}$ communications:

\section{A. - Transmitting thread:}

1. Data is read from the DGPS system and 5 variables are initialized: Timestamp, UTM_North, UTM_East, Speed and Direction.

2. Instants for starting broadcast transmission within control cycle are calculated as follows: $m$ independent values are taken from stochastic variable $X=U[0,1]$, giving values in increasing order to $X_{1}, \ldots, X_{m}$. Instants for $m$ transmissions will be: Time $_{i}[\mu \mathrm{s}]=$ Timestamp $[\mu \mathrm{s}]+160 \cdot \operatorname{int}\left(X_{i} \cdot 1250\right)$.

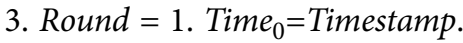

4. Pause until Time Round.

5. If int (Time Round_Timestamp)/40000> $\left(\right.$ Time $_{\text {Round }-1}$-Timestamp $) / 40000$, then speed is updated by reading information taken from the CAN bus. All fields in the next packet are updated consequently.

6. The broadcast packet is composed and sent at instant Time Round $_{\text {. }}$

7. Round $=$ Round +1 . If Round $<m+1$ then go to 4.

\section{B. - Receiving thread:}

1. Depending on the speed of the vehicle, variable $D$ is set up to the distance required by the vehicle to stop completely. Variable $E$ is set up to an increment in the distance considered necessary to be added to $D$ in order to account for the positioning error.

2. Packets are received at wireless interface. If a packet is the first one from a given station at this control cycle, the registry of that station is updated. If a packet is received from the station that already has sent the packet in this control cycle, it is thrown away.

3. When updated registry corresponds to a preceding vehicle, is received and if:

- deceleration is higher than $7 \mathrm{~m} / \mathrm{s}^{2}$;

- distance to our vehicle is less than $D+E+D G P S$ error +safety distance $(<15 \mathrm{~m})$;

- the emergency breaking system is activated to stop the vehicle.

4. At the end of control cycle:

- registries corresponding to the stations from which no packets have been received in the last three control cycles are erased;

- the number of registries is counted and assigned to variable $N$;

- if $N>40$, then transmission power is reduced to $3 \mathrm{dBm}$.

- $m$ is adjusted to the optimal value for $N$.

5. Go to 1 . 
Considering that any packet received from the neighbour's vehicle may contain positioning information with an error due to DGPS error plus an offset due to the estimation of updated position, Table 3 shows maximum positioning errors at different speeds

Table 3. Speed, distance covered and error in position

\begin{tabular}{cccc}
\hline $\begin{array}{c}\text { Speed } \\
(\mathrm{km} / \mathrm{h})\end{array}$ & $\begin{array}{c}\text { Speed } \\
(\mathrm{m} / \mathrm{s})\end{array}$ & $\begin{array}{c}\text { Distance Covered } \\
\text { in } 40 \mathrm{~ms}\end{array}$ & $\begin{array}{c}\text { Maximum error } \\
\text { in position }(\mathrm{E})\end{array}$ \\
\hline 10 & 2.78 & 0.11 & 0.61 \\
\hline 20 & 5.56 & 0.22 & 0.72 \\
\hline 30 & 8.33 & 0.33 & 0.83 \\
\hline 40 & 11.11 & 0.44 & 0.94 \\
\hline 50 & 13.89 & 0.56 & 1.06 \\
\hline 70 & 19.44 & 0.78 & 1.28 \\
\hline 90 & 25.00 & 1.00 & 1.50 \\
\hline 120 & 33.33 & 1.33 & 1.83 \\
\hline
\end{tabular}

The vehicle has to know maximum distance required to stop the vehicle (D) at a given speed and maximum error in position (E). When using this information, the previous algorithm stops the vehicle without collision as soon as a preceding vehicle reduces too abruptly its distance to our vehicle and its speed.

The V2V communication system has been widely tested in many trials in CAR facilities with only two vehicles. The presence of a packet injector in the scenario represents the effect of traffic generated by other contending vehicles. During these trials, the worst case of communication failure detected was the loss of signal during one control cycle.

\section{Experimental Results}

Various trials were performed to test the behaviour of the system. Fig. 11 shows a plan view of the private driving circuit at CAR facilities. This circuit represents an urban area with a combination of straight-road segments, bends and $90^{\circ}$ crossroads. The main aim of the present trials was to test the behaviour of the emergency braking system. The route selected to carry them out is marked in the figure. During the first four stretches, the autonomous car was performing ACC. The upper part of the circuit was used for the braking test. It consists of a

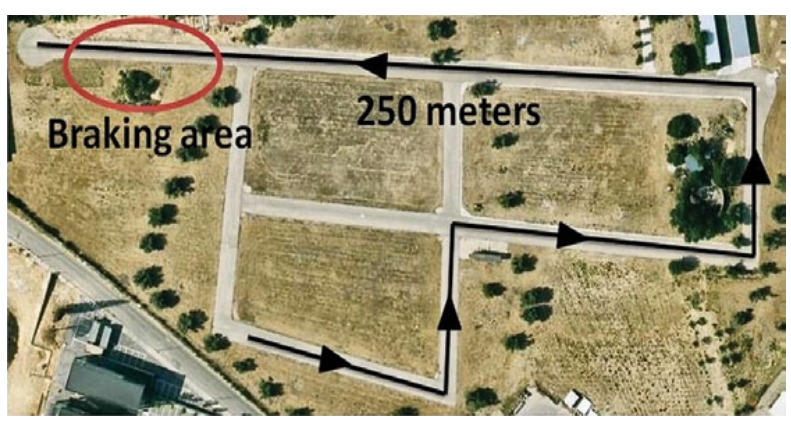

Fig. 11. Private driving circuit in CAR facilities straight $250 \mathrm{~m}$ stretch of the road, the last part (marked on the image) of which has a $3 \%$ downwards slope. We used this area for checking the system under the most unfavourable conditions of the circuit.

Two experiments will be described in detail. The first one was carried out with only one occupant in the car. It was made to check the behaviour of the system in case of losing the precision of the GPS signal - when IMU replaces GPS to determine a position. The second experiment was made with five occupants, so as to check the behaviour of the system in situations of different dynamics. The speed during these trials was around $50 \mathrm{~km} / \mathrm{h}$. The system was validated for speeds up to 55 $\mathrm{km} / \mathrm{h}$ because limitations on the circuit hindered from testing at higher speeds.

During these trials, the car was driven automatically to reduce human influence. It was accelerated from the starting point. When the car used to detect the manually driven preceding car, its speed was adapted until reaching the braking area where the preceding car executed a hard braking manoeuvre in order to test the operation of the new system. The preceding car was equipped with DGPS with the accuracy of up to $50 \mathrm{~cm}$ while the autonomous car was equipped with the RTK-DGPS system with the accuracy of up to $2 \mathrm{~cm}$ in order to achieve the best precision for graphical results. The proposed V2V communication system was implemented and used in the cars. Communication systems were Proxim Orinoco Gold cards working at the $5.8 \mathrm{GHz}$ band. The communication system itself was not stressed due to a low number of vehicles involved in the experiments. Hence, communications performed transparently as expected.

The goal of the first trial was to test the behaviour of our system when the failure of GPS occurred. To this end, software was modified to activate the IMU system even without the loss of GPS signal quality. In that way, we were able to compare the distance between the vehicles from GPS data with the positions generated by the IMU system.

Fig. 12 shows experimental results with one occupant in the car and a failure in GPS positioning. The top graph shows the speed of the leading (black) and trailing (grey) cars. The next one depicts the distance between cars - the black line shows the actual distance measured by GPS while the grey one indicates the distance when the position of the trailing car is generated by IMU. The following graph points to the acceleration of the leading car. These two values are input conditions needed to activate emergency braking. The bottom graph shows the value of the driving state variable (top black line) that selects different driving controllers the possible value of which are 0 for normal driving, 1 for ACC+Stop\&Go driving, 2 for overtaking, and 3 for emergency braking. In these experiments, only ACC+Stop\&Go driving and emergency braking are selected. The figure also shows the value of the threshold variable (bottom grey line) the values of which are 0 for normal state, -1 for the first trigger condition that the emergency stop has been satisfied (acceleration greater than $-7 \mathrm{~m} / \mathrm{s}^{2}$ ) and -2 indicates that both conditions (acceleration and distance) have been satisfied. 

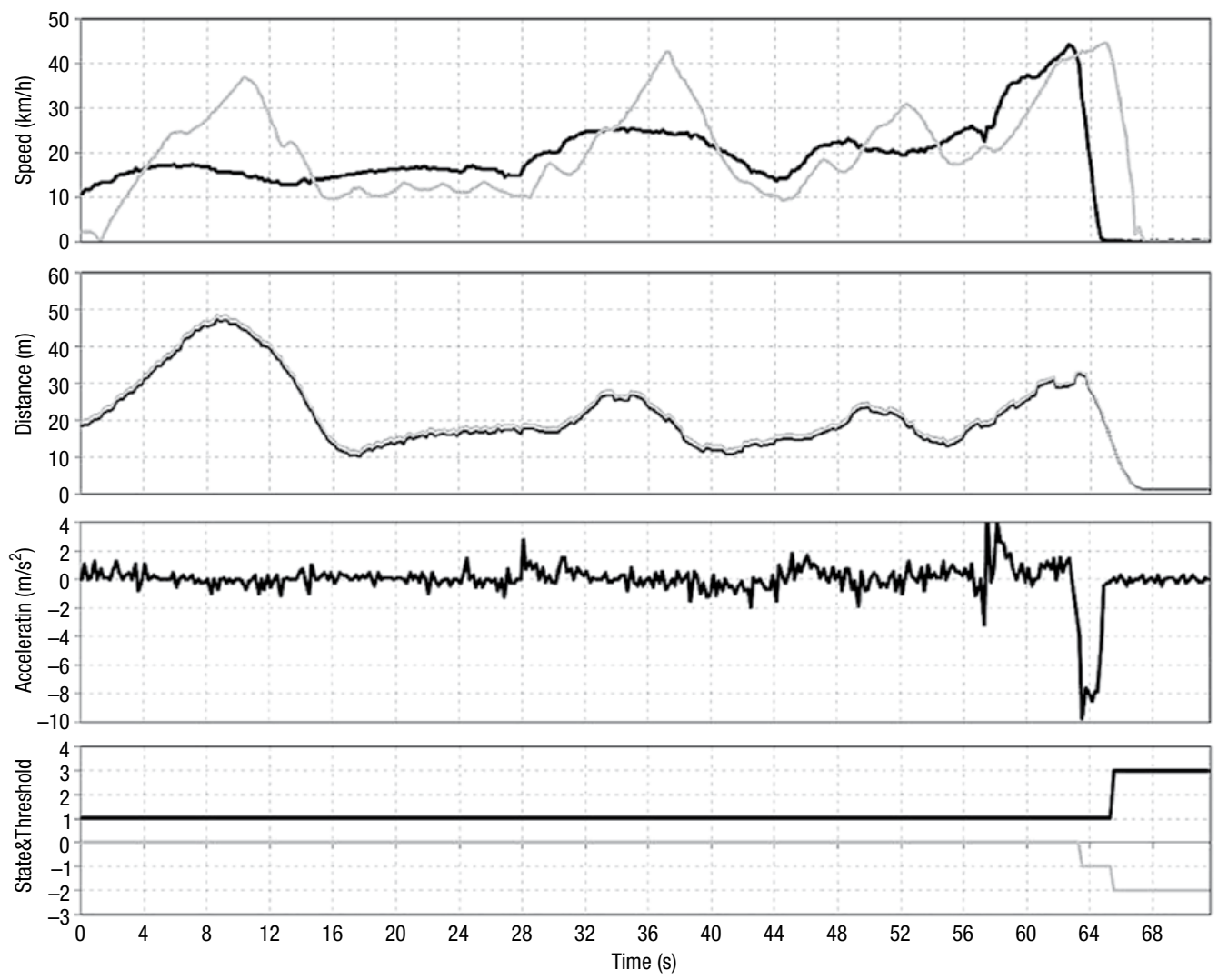

Fig. 12. Emergency braking experiment with one occupant and the IMU system

Initially, an autonomous vehicle follows the other one in the ACC+Stop\&Go driving state along the circuit depicted in Fig. 11. During the first $60 \mathrm{~s}$, the leading vehicle modifies its speed smoothly as shown in the acceleration graph. Next, following $63 \mathrm{~s}$, the driver of the leading car executes a hard braking manoeuvre for more than $2 \mathrm{~s}$ with deceleration values greater than $7 \mathrm{~m} / \mathrm{s}^{2}$ and a peak deceleration of $8.61 \mathrm{~m} / \mathrm{s}^{2}$. At this moment, the autonomous car is still in the ACC+Stop\&Go driving state.

Changes in the threshold variable are shown at the bottom part of Fig. 12. One observes that acceleration condition is fulfilled just before second 64, but the distance is then still greater than $15 \mathrm{~m}$. Soon after second 66 , the distance is reduced to less than this value, and then the threshold value changes to -2 thus triggering emergency brake. Therefore, the driving state value becomes 3 . The emergency brake system was activated and the trailing vehicle decelerated from $50 \mathrm{~km} / \mathrm{h}$ to zero in $15 \mathrm{~m}$.

Since inertial systems are dead-reckoning systems, their accuracy degrades with time. For instance, the use of inertial units causes the distance to be slightly greater than in reality so that the trigger of emergency brake is activated slightly earlier. The final gap between the cars measured by the IMU system was $84 \mathrm{~cm}$, though the actual distance measured by GPS made $1.16 \mathrm{~m}$.

The degradation of positioning based on the IMU system is acceptable up to five minutes (Milanés et al.
2008), after which it becomes too large to be useful. In this trial, the duration of GPS outage was around 80 seconds.

The second trial consisted of repeating the same route but had five occupants in the autonomous car which was so as to assess the influence of the mass on the system. The results are shown in Fig. 13. In this case, the gap when the vehicles had finally stopped was $74 \mathrm{~cm}$.

To sum up, the conducted tests under unfavourable conditions of slope, positioning and load showed the system to present good behaviour.

We have only described one trial of each kind in unfavourable conditions. However, many different trials were actually carried out, and in all cases, the final distance between vehicles was less than $75 \mathrm{~cm}$ when GPS was working at its top precision, and $1.25 \mathrm{~m}$ in the case of GPS outage. Given that the manually driven car had DGPS with an associated error of around $50 \mathrm{~cm}$, the obtained results show the good behaviour of the system.

\section{Appraisal}

The proposed system focuses on emergency braking based on V2V communications in urban environments (speeds up to $50 \mathrm{~km} / \mathrm{h}$ ). Nevertheless, it is obvious that in order to develop a commercial system, a new variable would have to be introduced: deceleration and its value would have to depend on the road surface and meteorological conditions. 

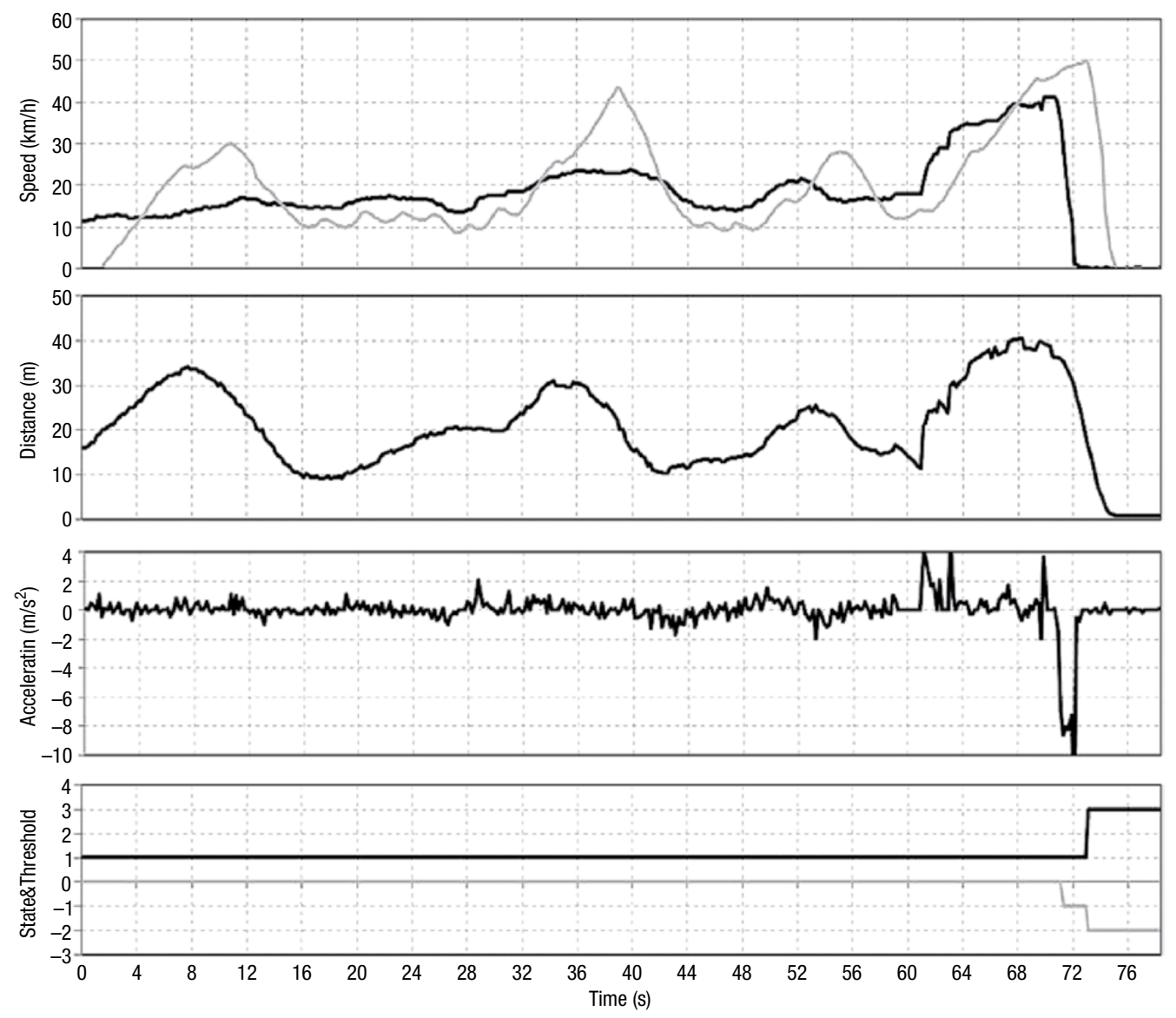

Fig. 13. Emergency braking experiment with five occupants

Everything this paper deals with is closely related to conditions on the road, namely, the friction coefficient between the tire and the road. There are many studies concerning this subject. Table 4 taken from (Wong 2008) shows friction coefficients taking into account the function of the surface. A new column has been added to show deceleration values in each one of these surfaces. As far as this work aims, only asphalt dry conditions have to be considered (deceleration higher than $7 \mathrm{~m} / \mathrm{s}^{2}$ ).

In order to improve the proposed system, a different coefficient would have to be selected according to

Table 4. Speed, distance covered and error in position

\begin{tabular}{lccc}
\hline \multicolumn{1}{c}{ Surface } & $\begin{array}{c}\text { Peak Value } \\
\mu_{p}\end{array}$ & $\begin{array}{c}\text { Sliding } \\
\text { Value } \mu_{S}\end{array}$ & $\begin{array}{c}\text { Peak } \\
\text { Acceleration } \\
\left(\mathrm{m} / \mathrm{s}^{2}\right)\end{array}$ \\
\hline $\begin{array}{l}\text { Asphalt and } \\
\text { concrete (dry) }\end{array}$ & $0.80 \div 0.90$ & 0.75 & $7.84 \div 8.82$ \\
\hline Asphalt (wet) & $0.50 \div 0.70$ & $0.45 \div 0.60$ & $5.39 \div 6.86$ \\
\hline Concrete (wet) & 0.80 & 0.70 & 8.84 \\
\hline Gravel & 0.60 & 0.55 & 5.88 \\
\hline Earth road (dry) & 0.68 & 0.65 & 6.66 \\
\hline Earth road (wet) & 0.55 & $0.40 \div 0.50$ & 5.39 \\
\hline Snow (hard-packed) & 0.20 & 0.15 & 1.96 \\
\hline Ice & 0.10 & 0.07 & 0.98 \\
\hline
\end{tabular}

weather conditions. It could be obtained from the CAN bus, i.e. using information coming from windshield wipers for rain conditions.

In brief, the proposed system achieves the initial goals but further work is necessary if a robust commercial system is to be obtained. V2V communications have been deeply discussed. Next steps in road conditions and its effects on the proposed algorithm - both trigger and controller - have to be acknowledged.

\section{Conclusions}

1. A system based on V2V communication and electrohydraulic braking to take over control of the car in the case of an unexpected situation has been presented. This system has been incorporated into the architecture of AUTOPIA autonomous driving control. DGPS signal was used for determining distances between cars, and the method of precise stopping was designed and implemented to stop the car before a collision could occur. The system was designed to have collision avoidance as its primary target.

2. Different experimental trials were performed to check the behaviour of the system giving good results. The errors obtained were less than $1.25 \mathrm{~m}$ under really unfavourable conditions, showing that such design is a hopeful starting point for developing automatic devices that will act on the cars independently of the driver when a possible accident is detected by some 
means and all warnings have failed to produce driver's response.

3. As works for the future, effects on changes under road conditions have to be considered. They affect both the trigger system - deceleration values lower than $7 \mathrm{~m} / \mathrm{s}^{2}$ have to be considered - and the controller. The proposed algorithm will have to take into account DGPS error, communication failures and delays and a new variable that is the effect of the road adhesion coefficient.

\section{Acknowledgments}

The authors wish to express their gratitude to Spain's Ministerio de Fomento and Plan Nacional for supporting the GUIADE (P9/08) and TRANSITO (TRA200806602-C03-01) projects respectively without which this work could not have been carried out.

\section{References}

Amodeo, M.; Ferrara, A.; Terzaghi, R.; Vecchio, C. 2010. Wheel slip control via second-order sliding-mode generation, IEEE Transactions on Intelligent Transportation Systems 11(1): 122-131. doi:10.1109/TITS.2009.2035438

Anwar, S.; Zheng, B. 2007. An antilock-braking algorithm for an eddy-current-based brake-by-wire system, IEEE Transactions on Vehicular Technology 56(3): 1100-1107. doi:10.1109/TVT.2007.895604

Boumediene, A.; Brahimi, K.; Belguesmia, N.; Bouakkaz, K. 2009. Saturation flow versus green time at two-stage signal controlled intersections, Transport 24(4): 288-295. doi:10.3846/1648-4142.2009.24.288-295

Bu, F.; Tan, H.-S. 2007. Pneumatic brake control for precision stopping of heavy-duty vehicles, IEEE Transactions on Control Systems Technology 15(1): 53-64. doi:10.1109/TCST.2006.883238

Çalışkanelli, P.; Özuysal, M.; Tanyel, S.; Yayla, N. 2009. Comparison of different capacity models for traffic circles, Transport 24(4): 257-264. doi:10.3846/1648-4142.2009.24.257-264

Černiauskas, E.; Keršys, A.; Lukoševičius, V.; Sapragonas, J. 2010. Investigation of anti-intrusion beams in vehicle side doors, Mechanika (6): 11-16.

Djukić, M.; Rusov, S.; Mitrović, Z. 2010. A fuzzy model for an increase in locomotive traction force, Transport 25(1): 36-45. doi:10.3846/transport.2010.06

Gerdes, J. C.; Hedrick, J. K. 1997. Vehicle speed and spacing control via coordinated throttle and brake actuation, Control Engineering Practice 5(11): 1607-1614. doi:10.1016/S0967-0661(97)10016-8

Grīslis, A. 2010. Longer combination vehicles and road safety, Transport 25(3): 336-343. doi:10.3846/transport.2010.41

IEEE Std 802.11:2007. IEEE Standard for Information Technolo$g y$-Telecommunications and Information Exchange Between Systems-Local and Metropolitan Area Networks-Specific Requirements - Part 11: Wireless LAN Medium Access Control (MAC) and Physical Layer (PHY) Specifications.

IEEE P802.11p/D3.0:2007. Draft Amendment to Standard for Information Technology-Telecommunications and Information Exchange between Systems-Local and Metropolitan Area Networks-Specific Requirements - Part 11: Wireless
LAN Medium Access Control (MAC) and Physical Layer (PHY) Specifications-Amendment 7: Wireless Access in Vehicular Environment.

Iyer, A.; Kherani, A.; Rao, A.; Karnik, A. 2008. Secure V2V communications: Performance impact of computational overheads, in 2008 IEEE INFOCOM Workshops: Conference on Computer Communications, 1-6. doi:10.1109/INFOCOM.2008.4544660

Jiang, D.; Delgrossi, L. 2008. IEEE 802.11p: towards an International Standard for WAVE, in 2008 IEEE 67th Vehicular Technology Conference-Spring, 2036-2040. doi:10.1109/VETECS.2008.458

Junevičius, R.; Bogdevičius, M. 2009. Mathematical modelling of network traffic flow, Transport 24(4): 333-338. doi:10.3846/1648-4142.2009.24.333-338

Kim, H. M.; Dickerson, J.; Kosko, B. 1996. Fuzzy throttle and brake control for platoons of smart cars, Fuzzy Sets and Systems 84(3): 209-234. doi:10.1016/0165-0114(95)00326-6

Milanés, V.; Naranjo, J. E.; González, C.; Alonso, J.; de Pedro T. 2008. Autonomous vehicle based in cooperative GPS and inertial systems, Robotica 26(5): 627-633. doi:10.1017/S0263574708004232

Naranjo, J.E.; González, C.; García, R.; de Pedro T. 2006. ACC+Stop\&Go maneuvers with throttle and brake fuzzy control, IEEE Transactions on Intelligent Transportation Systems 7(2): 213-225. doi:10.1109/TITS.2006.874723

Prentkovskis, O.; Beljatynskij, A.; Juodvalkienè, E.; Prentkovskiene, R. 2010a. A Study of the deflections of metal road guardrail post, The Baltic Journal of Road and Bridge Engineering 5(2): 104-109. doi:10.3846/bjrbe.2010.15

Prentkovskis, O.; Sokolovskij, E.; Bartulis, V. 2010b. Investigating traffic accidents: a collision of two motor vehicles, Transport 25(2): 105-115. doi:10.3846/transport.2010.14

Prohaska, R.; Devlin, P. 1998. Combined Brake and Steering Actuator for Automatic Vehicle Control. California Partners for Advanced Transit and Highways (PATH). California PATH Working Paper UCB-ITS-PWP-98-15. 18 p. Available from Internet: <http://www.path.berkeley.edu/path/publications/ pdf/PWP/98/PWP-98-15.pdf $>$.

Sapragonas, J.; Dargužis, A. 2011. Model of radial deformations of protector of vehicle tire, Mechanika 17(1): 21-29.

Sen, I.; Matolak, D. 2008. Vehicle-vehicle channel models for the 5-GHz band, IEEE Transactions on Intelligent Transport Systems 9(2): 235-245. doi:10.1109/TITS.2008.922881

Skrickij, V.; Bogdevičius, M. 2010. Vehicle gearbox dynamics: centre distance influence on mesh stiffness and spur gear dynamics, Transport 25(3): 278-286. doi:10.3846/transport.2010.34

Sokolovskij, E.; Prentkovskis, O.; Pečeliūnas, R.; KinderytèPoškienè, J. 2007. Investigation of automobile wheel impact on the road border, The Baltic Journal of Road and Bridge Engineering 2(3): 119-123.

Tan, H.-S.; Huang, J. 2006. DGPS-based vehicle-to-vehicle cooperative collision warning: engineering feasibility viewpoints, IEEE Transactions on Intelligent Transportation Systems 7(4): 415-428. doi:10.1109/TITS.2006.883938

Tufail, A.; Fraser, M.; Hammad, A.; Kim, K. H.; Seung-Wha, Y. 2008. An empirical study to analyze the feasibility of WIFI for VANETs, in 12th International Conference on Computer Supported Cooperative Work in Design, 553-558. doi:10.1109/CSCWD.2008.4537038 
Use of Intelligent System in Vehicles. 2006. European Commission. 109 p. Available from Internet: <http://ec.europa.eu/ public_opinion/archives/ebs/ebs_267_en.pdf>.

Uzcategui, R.; Acosta-Marum, G. 2009. WAVE: A Tutorial, IEEE Communications Magazine 47(5): 126-133. doi:10.1109/MCOM.2009.4939288

Venture, G.; Ripert, P.-J.; Khalil, W.; Gautier, M.; Bodson, P. 2006. Modeling and identification of passenger car dynamics using robotics formalism, IEEE Transactions on Intelligent Transportation Systems 7(3): 349-359. doi:10.1109/TITS.2006.880620

Wong, J. Y. 2008. Theory of Ground Vehicles. 4th edition. Wiley. $592 \mathrm{p}$.

Xiang, W.; Richardson, P. C.; Zhao, C.; Mohammad, S. 2008. Automobile brake-by-wire control system design and analysis, IEEE Transactions on Vehicular Technology 57(1): 138-145. doi:10.1109/TVT.2007.901895

Zhou, X.; Mahmassani, H. S. 2006. Dynamic origin-destination demand estimation using automatic vehicle identification data, IEEE Transactions on Intelligent Transportation Systems 7(1): doi:105-114. 10.1109/TITS.2006.869629

Zhuan, X; Xia, X. 2007. Optimal scheduling and control of heavy haul trains equipped with electronically controlled pneumatic braking systems, IEEE Transactions on Control System Technology 15(6): 1159-1166.

doi:10.1109/TCST.2007.899721 